\title{
Design and Implementation of a Scalable Constraint-based Routing Module for QoS Path Computation
}

\author{
Pablo Gainza \\ Escuela de Ciencias de la \\ Computacion e Informatica \\ Universidad de Costa Rica, \\ San Pedro, Costa Rica \\ pgainza@ecci.ucr.ac.cr
}

\author{
Hector Cancela \\ Instituto de Computacion \\ Universidad de la Republica \\ Montevideo, Uruguay \\ cancela@fing.edu.uy \\ Maria E. Urquhart \\ Instituto de Computacion \\ Universidad de la Republica \\ Montevideo, Uruguay \\ urquhart@fing.edu.uy
}

\author{
Eduardo Grampin \\ Instituto de Computacion \\ Universidad de la Republica \\ Montevideo, Uruguay \\ grampin@fing.edu.uy
}

\begin{abstract}
Quality of Service in computer networks depends on several variables like bandwidth, delay, jitter, cost, and packet loss which affect the overall user experience. In Multiprotocol Label Switching Networks, paths can be established end-to-end and resources can be guaranteed on each hop. However, due to several reasons, among them the computational cost involved, these paths are normally computed with constraints for only one variable. In this paper we propose a design and implementation of a scalable module for multi-constraint path computation in the context of the Path Computation Element architecture. We present all the design issues involved in an integral solution that accounts for all problems involved, some examples of the problem, and a scalable architecture to solve it. Finally, we present our performance results on simulated scenarios and real world networks.
\end{abstract}

\section{Categories and Subject Descriptors}

C.4 [Performance of Systems]: Design studies

\section{General Terms}

Algorithms design experimentation

\section{Keywords}

Protocols and Routing, Optical Networks

\section{INTRODUCTION}

In the past few years, the Multiprotocol Label Switching Protocol (MPLS) has become a competitive alternative for

Permission to make digital or hard copies of all or part of this work for personal or classroom use is granted without fee provided that copies are not made or distributed for profit or commercial advantage and that copies bear this notice and the full citation on the first page. To copy otherwise, to republish, to post on servers or to redistribute to lists, requires prior specific permission and/or a fee.

LANC'07 October 10-11, San Jose, Costa Rica.

Copyright 2007 ACM 978-1-59593-907-4/07/0010 ...\$5.00. the establishment of end-to-end paths on legacy IP infrastructure. These paths, called Label Switched Paths (LSP), allow the reservation of resources along every involved node, acting like a dedicated line, which allows the protocol to guarantee certain Quality of Service (QoS) requirements, making it a promising technology. The recent RFC 4655 calls for the creation of a an outside entity, the Path Computation Element, to manage path computation, signalling and topology database management. Furthermore, the Generalized MPLS Standard calls for the dynamic creation of paths from source to destination in an MPLS networks, which would allow, for instance, the creation of an LSP guaranteeing certain QoS requirements when a user dials a phone number, from the user to the destination.

QoS normally does not depend on only one variable (i.e. bandwidth), but on several ones, like for example, delay, delay jitter, and packet loss probability. The use of those variables, and more importantly, the constraints placed upon them, depends on the appplication and user perception: streamed video may tolerate a high delay and packet loss, but not a high jitter, while voice may not tolerate a high packet loss. The traditional algorithms for path computation, like Dijkstra or Bellman-Ford are ineffective when dealing with several constraints, and the exhaustive search algorithm for the solution space is too computationally expensive.

Several heuristics and exact algorithms have been presented to solve the problem of multi-constrained paths (MCP) and multi-constrained optimal paths (MCOP), and with increased computational power, it is now possible to implement them on a real network. Kuipiers et al in [14] present an extensive review of these algorithms, and in [16], [18] present algorithms to related problems. [13], [21], and [23] propose other algorithms for the $\mathrm{MCP} / \mathrm{MCOP}$ problem. The introduction of the PCE has also taken the computational burden away from the communications hardware, to an arbitrarily powerful dedicated machine. In this respect we follow the ideas of [7], which proposes an implementation of the PCE.

However, several questions remain as to the real feasibility of computing paths with multiple constraints, to its performance, and scalability. To our knowledge, the only work 
which deals with an integral solution to the Constraint-based routing problem is in [6]. Unfortunately, the solution was not implemented and tested and we believe it lacks scalability due to the algorithms chosen.

The goal of this work is to design and implement an integral PCE component that performs path computation subject to multiple constraints, which we will call the Constraintbased Routing module (CBR), as proposed in [9], to evaluate its feasibility, and to measure its performance and scalability in simulated and real world scenarios. The role of the CBR is to receive a path request from a source node to a destination node with a series of constraints, extract the topology from the Traffic Engineering Database (TED) and return a path that satisfies both the constraints and other requirements. This component is only concerned with the path computation; the TED is maintained by other components, and the path(s) produced by this component are sent to the path signaling component.

We aim at having this module manage all path computation inside a medium-sized network in the context of the PCE, to do it in a very small time, and to be able to handle any attribute the administrator defines, and any constraint the client requests over those attributes.

This paper is organized as follows. Section 2 presents a description of the problem and a formal definition. Section 3 presents the design issues involved in the decision and the chosen algorithms to tackle them. On section 4 we present a scalable architecture to address the problem and the algorithms for it. Finally, on section 5 we present performance results for the online part of the CBR.

\section{THE PROBLEM}

QoS constraints can be divided in two categories: additive/multiplicative constraints, that involve the whole path, such as delay, hop count, loss probability or cost; and nonadditive constraints, also called minimum(maximum), that require each link or node to meet certain bounds, such as bandwidth, proccessing load or administrative decisions. To solve the nonadditive constraints problem, a topology filtering algorithm[4] is necessary to filter out all links and nodes that do not satisfy $\min (\max )$ bounds from the topology.

On the other hand, finding a path that satisfies multiple additive/multiplicative constraints is known to be an NPHard problem. Figure 1 presents an architecture to illustrate how the MCOP problem is NP-hard. Imagine that a path must be established between $\mathrm{S}$ and $\mathrm{D}$ obeying constraints: Delay $\leq 10$ and Loss probability $\leq 4 \%$, while optimizing cost. With only one variable (i.e. Cost), the Dikjstra Algorithm can find a solution in $O(n)$ time. With more than one variable, all permutations must be tested to find the optimal path, since there is no "best path" from $S$ to 2 , or from 2 to $D$. A formal proof can be found in [19].

The PCE imposes several additional requirements that are not normally dealt with by these solutions. The PCE computes paths online, which imposes a constraint on the amount of time that the algorithm may take, which, in the worst case, is proportional to its complexity. Since the PCE can be scaled to meet its processing requirements, the parallelization of the algorithm is highly desired. In addition, there are several cases where the implementation must be able to process a set of paths, namely: when $\mathrm{S}$ sub-paths are explicitly requested; when backup, disjoint paths are requested; and in cases where a path with the requested

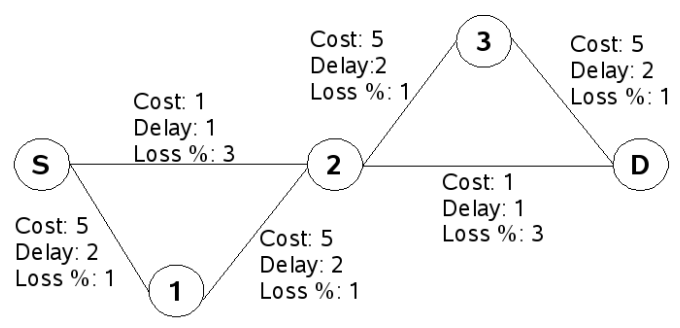

Figure 1: An example of the MCOP problem.

nonadditive constraints (i.e. bandwidth) is not found. Finally, path calculation must be optimized to fulfill traffic engineering objectives, which basically are to maximize network utilization and minimize path setup rejection.

Our work relies upon the assumption that maximum, and precise bounds for QoS constraints can be established. ${ }^{1}$

\subsection{Definitions and notation}

We now introduce a detailed definition for the MCP and MCOP. Our notation is based on [14] and [21]. This notation will be used throughout all the paper, where we will usually suppose that we will be given as input the network topology and a constrained path request.

\section{Definition 1.}

QoS Graph

Let $G^{\prime}=\left(N^{\prime}, E^{\prime}\right)$ be an directed graph that represents the current network topology and is extracted from the TED. $N^{\prime}$ are the nodes of this topology and $E^{\prime}$ are the edges. Each node $u$ and edge $(u, v)$ in the TED is characterized by a set of attributes, $A(u)$ and $A(u, v)$, that, as stated before, are gathered from the network.

In $G^{\prime}$, the nodes and edges will not be characterized by $A$, but by weight vectors. Each node $u$ will be characterized by a vector of nonadditive $\min (\max )$ weights, $w^{N}(u), u \in N$ of size $\left|w^{N}\right|$, where $w_{i}^{N}(u)=f_{i}^{N}(A(u))$ and $f_{i}^{N}$ is a programmed function. Each edge will also be characterized by a vector of nonadditve weights, $w^{E}(u, v),(u, v) \in E$ of size $\left|w^{E}\right|$, where $w_{i}^{E}(u, v)=f_{i}^{E}(A(u, v))$ and $f_{i}^{E}$ is a programmed function.

For additive/multiplicative weights for nodes and edges, there will be one vector, $w^{A}(u, v),(u, v) \in E$ where $w_{i}^{A}(u, v)=$ $f_{i}^{A}(A(u), A(v), A(u, v)) \geq 0$. For simplicity, multiplicative weights are converted using logarithms to possitive, additive values ${ }^{2}$ and weights involving node attributes are condensed into one edge weight (i.e. $w_{i}^{A}(u, v)=w_{i}^{A}(u)+w_{i}^{A}(v)$ for additive node weights).

In addition, all nonadditive weights max weights will be converted into min weights, by multiplying both weights and (later to be defined) constraints by -1 . This means that from now on we will only be looking at additive and nonad-

\footnotetext{
${ }^{1}$ Instead of working with the measured value of an attribute, we could work with probabilistic upper and lower bounds for that attribute, since attributes like delay or jitter change depending on external factors. We could, for instance, define an attribute $a$ such that $p\left(a_{i} \leq a\right) \geq \alpha$, where $\alpha$ is an

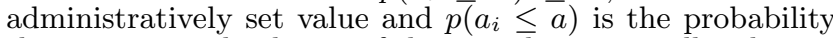
that a measured value $a_{i}$ of that attribute is smaller than $a$. ${ }^{2} \mathrm{~A}$ detailed explanation of the use of logarithms to convert multiplicative weights to additive ones can be found in [15]
} 
ditive min weights.

\section{Definition 2.}

Constrained path request

The constrained path request consists of a source node, $s$; a destination node, $d$; and the constraints that represent the values that will be compared against the weights. $C^{N}$, of size $\left|w^{N}\right|$ will be the vector that represents $\min (\max )$ values of $w^{N} ; C^{E}$, of size $\left|w^{E}\right|$ for $w^{E}$; and $C^{A}$ of size $\left|w^{A}\right|$ will represent maximum values for the sum of the weights $w^{A}$ in a path. Colored resources, which are the resources through which the path may or may not pass can be represented as nonadditive constraints. Finally, a constrained path request may contain a backup path request. We define this with a flag, B.

Therefore, we define a Constrained path request $R$ as:

$$
R=\left\{s, d, C^{N}, C^{E}, C^{A}, B\right\}
$$

\section{Definition 3.}

\section{Filtered topology}

We can now define a filtered topology, $G=(N, E)$, that purges all the nodes and edges that do not meet the min(max) bounds.

$$
N=\left\{u / u \in N^{\prime} \wedge w_{i}^{N}(u) \leq C_{i}^{N} \text { for } i=1, \ldots,\left|C^{N}\right|\right\}
$$

The edge set, $E$, is the result of purging not only edges not meeting the required bounds but also all edges whose end nodes are not in $N$.

$$
\begin{array}{r}
E=\left\{(u, v) /(u, v) \in E^{\prime}, u, v \in N \wedge\right. \\
\left.w_{i}^{E}(u, v) \leq C_{i}^{E} \text { for } i=1, \ldots,\left|C^{E}\right|\right\}
\end{array}
$$

\section{Definition 4.}

Multiconstrained path Let $P$ be a path for a Path Request $R$ from $s$ to $d$ where:

$$
w_{i}^{A}(P)=\sum_{(u, v) \in P} w_{i}^{A}(u, v)
$$

Let $m=\left|C^{A}\right|$. By definition, $w^{A}(p)=\left(w_{1}^{A}, \ldots, w_{m}^{A}\right)$. We say that $\mathrm{P}$ is a Multiconstrained path if

$$
w_{i}^{A}(P) \leq C_{i}^{A} \text { for } i=1, \ldots, m
$$

\section{Definition 5.}

Multiconstrained optimal path We say that a path $P$ is a multiconstrained optimal path if:

$$
l(P) \leq l\left(P^{*}\right) \forall P^{*}, P \text { satisfying Definition } 4 .
$$

where $l(P)$ can be a linear or nonlinear cost function of the weights of $P$, such as the one proposed by Korkmaz [13]. In our case, $l(P)$ will aim to maximize network utilization and minimize LSP setup rejection.

\section{Definition 6.}

Multiconstrained optimal backup path

We define a backup path, $P^{*}$, whose edges are different from those of $P$. Even though backup paths may be defined using administrative constraints, which would be more flexible, it is much more efficient to compute them together.
Let $P$ and $P^{\prime}$ be two multiconstrained paths (Definition 4) from $s$ to $d$. We say that $P^{\prime}$ is a multiconstrained optimal backup path of $P$ if:

(i) $\operatorname{Links}(P) \bigcap \operatorname{Links}\left(P^{\prime}\right)=\emptyset$

(ii) $\left(l(P)+l\left(P^{\prime}\right)\right) \leq\left(l\left(P^{*}\right)+l\left(P^{* \prime}\right)\right) \forall P^{*}, P^{* \prime}$ satisfying Definition 4

such that $\operatorname{Links}\left(P^{*}\right) \bigcap \operatorname{Links}\left(P^{* \prime}\right)=\emptyset$ and the function $\operatorname{Links}(P)$ is the set of all edges that are visited by path $P$. Note that when calculating disjoint backup paths, $P$ and $P^{\prime}$ are not necessarily optimal paths by their own, but jointly they should be the optimal pair.

\section{CBR DESIGN}

In this section we present the different aspects we had to tackle in order to design our proposed Online CBR. The main design issues are the following ones:

\section{Online CBR/Response Time:}

The PCE was conceived as an element which performs path computation online, as opposed to offline, which precalculates externally all the LSPs in a network according to a traffic demand matrix. In online TE, the path calculation must not only be made on a small amount of time to reduce connection setup, but it must also attempt to maximize network utilization and avoid blocking other path requests, without knowing what those path requests may be beforehand.

\section{Traffic engineering:}

The goal of traffic engineering is to maximize network throughput. If paths are established disregarding TE, the network's load will become unbalanced and links will become congested. To avoid this, link costs should be set with the objective of achieving load balancing, avoiding congestion and minimizing blocking of other LSPs.

\section{Multi-constrained optimal paths:}

Since the above mentioned issue requires the optimization of a path cost, an algorithm that only resolves the multi-constrained path problem is not enough. A multiconstrained optimal path (Definition 5) should be able to optimize the Traffic engineering cost function.

\section{Disjoint QoS Paths:}

Finding two disjoint paths that satisfy the MCOP problem can be done in a simple way by finding the first path, pruning all the links of the first one from the graph and then finding the second one. However, by this method, it is not guaranteed that two disjoint paths that satisfy the constraints can be found, even if they exist; and if found, it is not guaranteed that they will be optimum.

\section{Job paralelization:}

The PCE, as an external entity, must be able to scale well in order to minimize response time and handle large networks.

In the following subsections we tackle these design issues separately; in Section 4 we present the proposed architecture, integrating the different solution elements. 


\subsection{Traffic engineering}

Obtaining an optimal distribution of LSPs with an offline path calculation of a traffic demand matrix is an NPComplete problem [3]. With online path computation the problem is exacerbated since future requests are not known.

Several algorithms have been proposed to try to avoid blocking other LSPs. Widest-shortest path [1] finds the shortest paths with the largest available bandwidth. MIRA [12] assigns a cost to each link according to its criticality. This value computed by finding the max-flow (using available bandwidth) between all pairs of ingress-egress routers. Simple MIRA [11] reduces the complexity of MIRA, which in the worst case is $O\left(n^{5}\right)$ by using $k$ shortest paths between each ingress and egress node instead of all. Even though Simple MIRA reduces the complexity of MIRA significantly, it is still relatively high, $O\left(n^{2}\right)$. These two algorithms are not apt for an online CBR process since each path calculation would have this complexity.

Another algorithm, Profile Based Routing (PBR) [22] assigns link costs associated with a traffic profile. A profile can be built in different ways, for example according to the SLAs or dinamically produced by observing traffic. PBR's complexity is reduced thanks to a preprocessing phase that solves the multi-commodity flow problem. However, PBR assumes that a traffic profile that will not change greatly through time can be built. Building the traffic profile is in itself a daunting task, and there may be cases where it cannot be built.

In [16], its authors propose SAMCRA-B an algorithm for network optimization to use with the multi-constrained optimal path problem that is similar to widest-shortest path first. However, SAMCRA-B differs in that below an administratively defined threshold, it routes LSPs through the minimum hop paths that satisfy the constraints. The goal of this threshold is to reduce the network resources used, SAMCRA-B uses the SAMCRA algorithm to compute paths.

DORA [2] improves MIRA's complexity by separating the process of assigning costs in two parts, one that calculates the Path Potential Value (PPV) using only disjoint paths between source and destination, and another that combines the PPV with the available bandwidth. The advantage lies in that the PPV has to be calculated only when the network topology changes, and can be done offline as a separate process. Therefore, its online complexity is $O(n)$.

\subsubsection{DORA pseudo code}

\section{Preprocessing Stage Process.}

Let $G^{\prime}=\left(N^{\prime}, E^{\prime}\right)$ be a graph representing the current network topology (Definition 1 ).

1. For each source-destination pair $(S, D)$ determine the set of all disjoint paths $D P_{(S, D)}$.

2. For each source-destination pair $(S, D)$, construct the $\operatorname{PPV}_{(S, D)}\left[\left|E^{\prime}\right|\right]$ array, and initialize all entries to zero.

3. For each source-destination pair $(S, D)$. For each link $l$ in the network, if $l$ is part of any path in $\mathrm{DP}_{(S, D)}$, substract 1 from $\operatorname{PPV}(S, D)[l]$. For all the source-destination pairs $\left(S^{\prime}, D^{\prime}\right),\left(S^{\prime}, D^{\prime}\right) \neq(S, D)$ inspect each link $l$ and determine the number of times $n$ that $l$ appears in $D P(S \prime, D \prime)$. Increment $\operatorname{PPV}_{(S, D)}[l]$ by $n$.

4. For each source-destination pair $(S, D)$, normalize all entries in $\mathrm{PPV}_{(S, D)}$, with the smallest PPV element over all source-destination pairs equal to 0 and the largest PPV el- ement over all source-destination pairs equal to 100 . Let $\operatorname{NPPV}_{(S, D)}[l]$ be equal to the normalized value of $\operatorname{PPV}_{(S, D)}[l]$.

\section{Online Weight Assignment.}

For a Path Request $R$

1. For each network link $l \in N$, extract its residual bandwidth $R B(l)$, and normalize $(R B(l))^{-1}$ to the range $0-100$, with the smallest $(R B(l))^{-1}$ value equal to 0 and the largest value equal to 100 . Let $N R B(l)$ be equal to the normalized value of $(R B(l))^{-1}$.

2. For the source-destination pair $(s, d)$, construct a link weight table $L W T_{(s, d)}$ using the following equation:

$L W T_{(s, d)}[l]=N P P V_{(s, d)}[l] \times(1-B W P)+N R B(l) \times(B W P)$

where BWP is the bandwidth proportion parameter. An interesting variation would be to use SAMCRA-B's threshold weight, where below a certain threshold, the link cost is the number of hops, which reduces resource usage.

We will choose DORA for online traffic engineering since it presents an online complexity of $O(n)$, and handles the potential value of a path. This path potential value is specially useful in asymetric networks or networks where not all nodes are source/destination (i.e. backbone nodes).

\subsection{Multi-constrained optimal paths}

Several heuristic algorithms as well as several exact ones have been proposed to address the MCP problem. A comprehensive review of these algorithms and a performance evaluation is presented in [14]. Among those proposed only a few are MCOP algorithms, H_MCOP, A*Prune and SAMCRA . H_MCOP [13] is the only heuristic of these algorithms, and presents the best execution time; since it is not an exact algorithm it can not guarantee optimal results. A*Prune is an exact algorithm that finds $k$ shortest paths between a source and a destination, but, its complexity growth can be exponential. SAMCRA is also an exact algorithm, based on four principles: nonlinear definition of the path length, a k-shortest path approach, nondominance and look-ahead. In [17], the authors prove that although the problem is NP-hard, the situations where this exact algorithm takes exponential time are very unusual and most likely would never be present on an actual network.

As a result, we will use SAMCRA as presented in [15], including two modifications. The first modification is to allow SAMCRA to handle directed graphs. We can achieve this by modifying the look-ahead function of SAMCRA to handle a reversed graph when searching for the distance from the end-node to each other node in the graph. The second modification it includes is a redefinition of the cost function, since SAMCRA's cost function minimizes the maximum weight of any of the constraints, while we aim to minimize the Traffic Engineering cost. Furthermore, the path request may specify a different weight for optimization, like hopcount, overriding the default TE cost, as described in the previous section. The new version is named SAMCRA_OP.

\subsection{Disjoint QoS paths}

The most intuitive way to find two node/link disjoint paths from a source to a destination is employing the RemoveFind (RF) method. This method consists of finding a shortest path, pruning the node/links of this path from the graph and finding the shortest path from the remaining part of the graph. This method, however, does not necessarily find two 
disjoint paths from source to destination in some cases where they actually exist; in other cases, even if it does find them, it does not guarantee that they satisfy Definition 6, which requires that the sum of their lengths be smaller than any other sum of lengths of shortest paths.

In traditional, one-dimensional, shortest path first routing, the augmenting path algorithm can find two disjoint paths in a graph by basically following these steps:

a. calculate the shortest path between source and destination

b. invert the direction of the links in the shortest path and multiply their weight by -1 .

c. recalculate the shortest path

d. Make a Union of the two paths and eliminate all the links whose inversed links are in the union. Make two paths out of the links that remain in the union.

However, in [18], Guo, Kuipers and their coauthors prove that this method does not apply to multiple dimensions since it can lead to loops. They therefore propose an alternative, called DIMCRA which which works in a similar way.

The DIMCRA algorithm, works well in solving the MCP problem, but it does not work with the MCOP problem. The reason is that it doubles the constraints before computing a partial path to handle cases where the partial path does not meet them, but the final path does. When the constraints are doubled, the SAMCRA cost function $w_{i}(P) / L_{i}$ guarantees that if a path is found that satisfies the constraints $2 L$, but does not satisfy the constraints $L$, no other path will satisfy them. When the cost function is modified to satisfy traffic engineering objectives, however, the same rule does not apply. Hence, the algorithm must be modified. We propose a modification, called DIMCRA_OP where the algorithm tries to find a path constrained by $L$, instead of $2 L$, and if none is found, DIMCRA is called, with the assumption that finding two paths that do not optimize cost is preferred over the alternative of not finding any path.

DIMCRA_OP $\left(G, C^{A}, s, t\right)$ : Given a directed graph $G=$ $(V, E)$, a constraint vector $C^{A}$, and a source-destination pair $(s, t)$ :

Step 1. Find the shortest path $\mathrm{P}_{1}$ obeying L with SAMCRA_OP; if $\mathrm{P}_{1}$ does not exist, then stop;

Step 2. Reverse the direction of all the links on the shortest path $\mathrm{P} 1$; and set the sign of their link weights zero, $w_{m}(v, u)=0 \forall(u, v) \in P_{1}$ and $m=1, \ldots, M$. A modified graph is created;

Step 3. Find the shortest path $P_{2}$ constrained by $L$ in the modified graph with SAMCRA_OP; if $P_{2}$ does not exist, then execute DIMCRA with $G=(V, E), C^{A}$, and $(s, t)$

Step 4. Make the union of $P_{1}$ and $P_{2}$, remove from the union the $P_{1}$ links whose reversed links appear on $P_{2}$; and vice versa, then group the remaining links into a set of two paths $\left\{P_{1}^{\prime}, P_{2}^{\prime}\right\}$

Step 5. Check the length of each path in the set $\left\{P_{1}^{\prime}, P_{2}^{\prime}\right\}$. If the path $P_{i}^{\prime}(1 \leq i \leq 2$ violates the constraints, then update the modified graph by removing the link set $P_{i}^{\prime}$ $\left(P_{i}^{\prime} \cap P_{i}\right)$ from it, and go to Step 3. Otherwise stop and return the current solution set $\left\{P_{1}^{\prime}, P_{2}^{\prime}\right\}$.

DIMCRA is a heuristic that not always returns a feasible path if one exists and it may return a non-optimal feasible path. Even though other proposals have been made

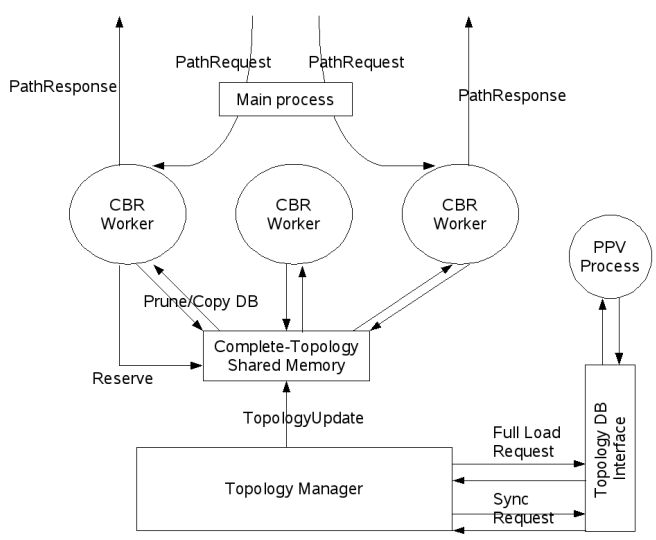

Figure 2: Architecture

[20] that attack the multi-constraint link/node disjoint path pair problem, they are limited to one constraint. The algorithm proposed [10] is much more succesful at calculating backup paths with multiple constraints (even though it consumes more resources) by using protected segments instead of whole disjoint paths. However, it requires that routers support these segments, which is out of the scope of the PCE proposal.

To our knowledge, DIMCRA is the only proposed algorithm that aims at finding a feasible set of disjoint paths restricted to $m$ constraints, making it the only one appropriate to attack our problem of a disjoint backup optimal path (Definition 6), even though it does not always comply with the definition.

\subsection{Job parallelization}

In order to provide for better scalability, the parallelization of online work is highly desired. However, the parallelization of the SAMCRA, and DIMCRA, was not part of their design and represents a daunting task. Therefore, our aim will be not to parallelize one instance of the algorithm, but to execute multiple instances in parallel, satisfying more than one request at a given time. We achieve this with a simple design that provides each instance with a fresh view of the TED and of the reserved resources.

\section{CBR ARCHITECTURE}

We now present our scalable architecture for the CBR.

Figure 2 shows a diagram of the architecture. In this proposal, a shared memory region will contain the Complete Topology and will be periodically updated from the Topology Database by the Topology Manager.

\subsection{A note on Resource consistency}

The PCE architecture presents a challenge regarding database consistency with the topology. When a PathResponse is produced, it is delivered to the signalling component, which sends an Explicit Route Object (ERO) to the destination LSR that will attempt to reserve the resources all the way back to the source path. If those resources are being used by another path, the path setup will fail and another request will be necessary. When all the resources have been reserved and the path established, an SNMP poll (or any 
other tool used to detect available resources) will extract the information from the network and refresh the database, which will eventually be synchronized with the Topology in shared memory.

The time $t$ that elapses from the moment the PathResponse is produced to the moment it is refreshed in the shared memory Topology could lead to a series of path calculations with resources that are no longer available. To solve this issue, we propose the creation of a set $U$ of assigned resources for each link and an associated timestamp. Since we are only dealing with one consumable resource, bandwidth, we define a resource estimated available bandwidth $E B$ :

$$
E B=B W-\sum_{\forall i} U_{i}
$$

where $B W$ is the available bandwidth as reported in the TED.

An element is added into the set $U$ of a link $l$ when a PathResponse $\mathrm{P}$ that contains $l$ is formed, adding the bandwidth of $\mathrm{P}$ and the timestamp.

An element is pruned from $U$ when an administratively defined time, $\gamma$, has elapsed since the element timestamp. The value of $\gamma$ could be defined, for example, as the maximum $t$.

$$
U=\{x / \operatorname{timestamp}(x)+\gamma<\text { time }\}
$$

Furthermore, when a Topology synchronization occurs and the value of the available bandwidth decreases by $b$ units, a subset $U^{\prime}$ of $U$ could be pruned from $U$, where:

(i) $\operatorname{timestamp}\left(U_{i}^{\prime}\right)<=\operatorname{timestamp}\left(\left\{U-U^{\prime}\right\}_{j}\right) \forall i, j$

(ii) $\sum_{\forall i} U_{i}<=b$

However, this last technique suggests that all bandwidth reservation is the product of a PathResponse by this PCE. If a bandwidth reservation occurs by means of another source (for example another PCE), resources that have just been reserved could be freed.

It is important to note that even if we reserve resources using these techniques, we cannot guarantee the knowledge of the actual resource availability, leading to cases where PathRequests are rejected when there are sufficient resources available and PathResponses are produced when there are no resources available.

\subsection{Main Process}

A Main Process will handle the initialization of the Topology Manager and of the processes that will do the actual computation (Algorithm 1).

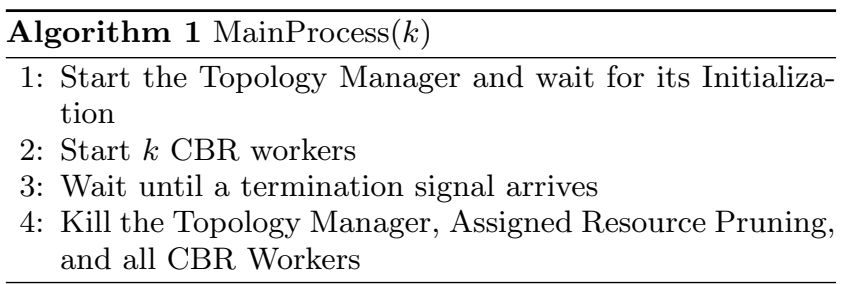

\subsection{Topology Manager}

The TED will be fully loaded initially by the Topology Manager and periodically refreshed using a sync operation
(Algorithm 2). The Topology Manager will also be in charge of pruning the assigned resources set as explained before.

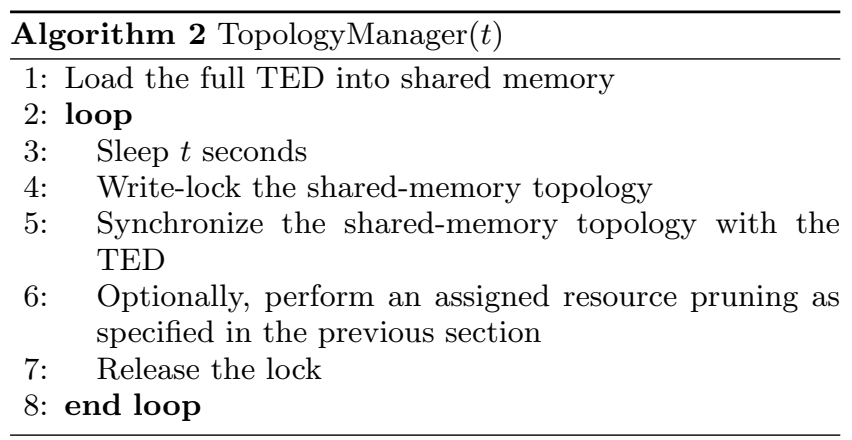

The assigned resource pruning algorithm (Algorithm 3) can be run inside the topology manager or on an outside process.

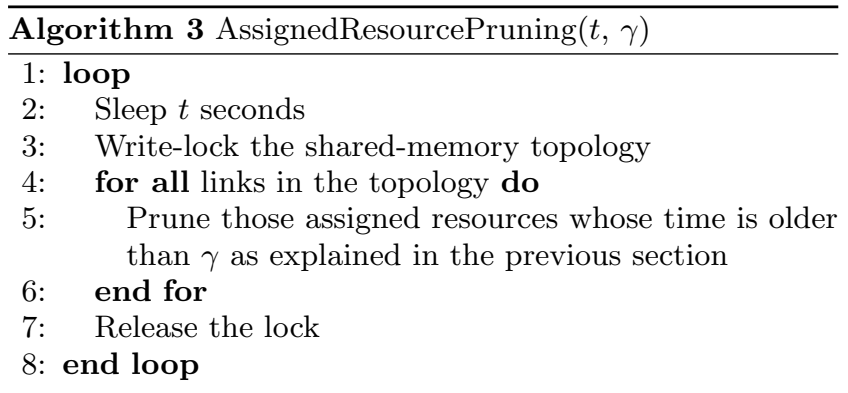

\subsection{CBR Worker}

The CBR worker (Algorithm 4) will be in charge of computing each subpath in the PathRequest with SAMCRA_OP (for a single path request) or with DIMCRA_OP (for a path plus backup path request). On each subpath computation, the CBR worker must perform a Topology Filtering and an Online Weight Assignment. After calculating a path, the worker must mark the used resources as assigned, as explained in the previous section. To do this it must lock write access to the shared memory region.

When two or more workers are performing a path calculation at the same time, there is a chance that both will use the same resources, and the last one to finish will not be able to reserve them. We will call this conflict resource contention; when it occurs, a path must be recalculated. Resource contention, and semaphore waiting could be the most significant overhead affecting the throughput of the parallel algorithm. Even if no resource contention occurs, two or more paths calculated concurrently can produce a negative effect on the network by unbalancing it, since the traffic engineering cost function depends on available bandwidth and it is unpredictable what effect the concurrent path computations will have on available bandwidth. We can reduce it by reserving a subpath's assigned resources immediately after it is calculated, as opposed to waiting until the whole PathRequest is calculated.

The proposed design could be used on a uniprocessor machine to allow simple path calculations to be served fast by an idle worker, even if a complex path calculation is taking place. This of course, with a drawback in throughput due to 


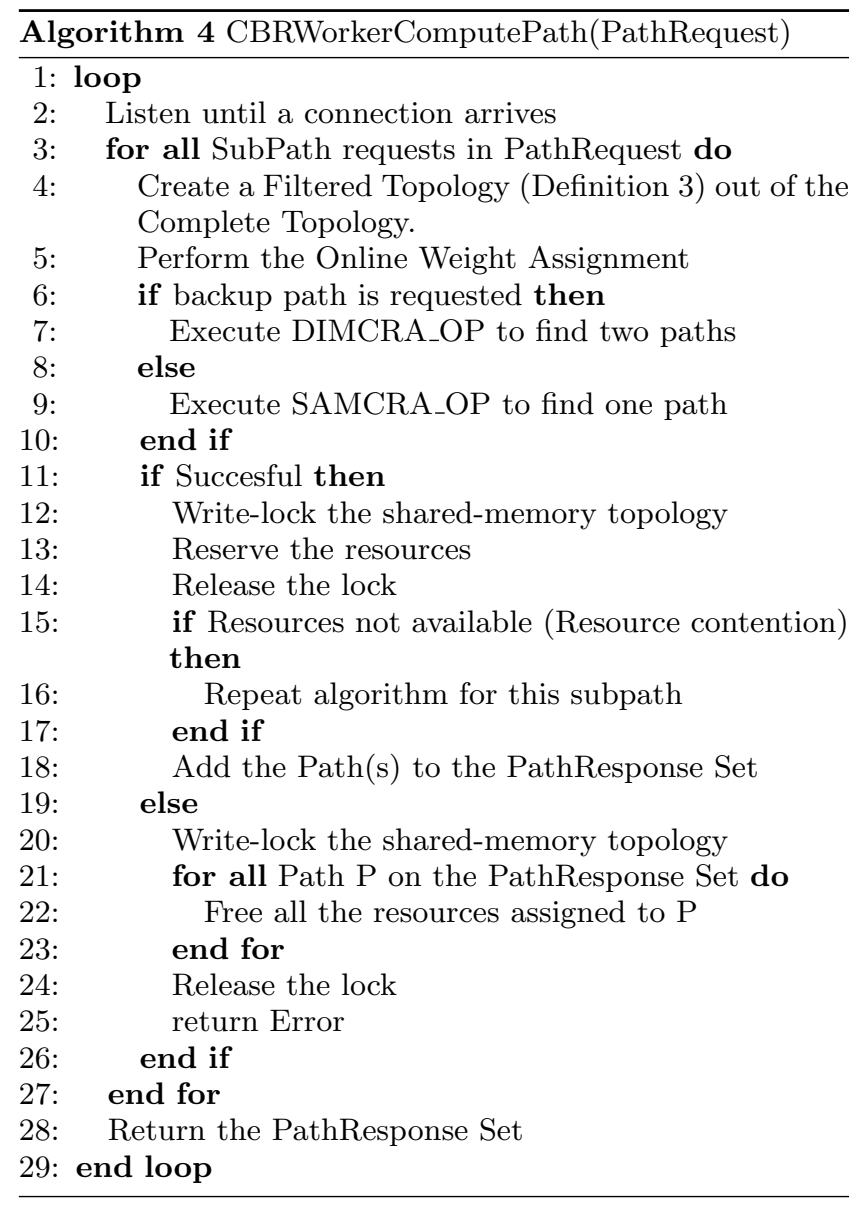

the overhead produced by semaphore waiting, resource contention, context switching, etc., when compared with the sequential alternative. On a multiprocessor machine, it could be used by having one or several workers per processor to improve throughput. On very large networks it could even be used on a distributed system by using distributed shared memory to maintain the topology on each worker.

On our case we will focus on the single memory, uni/multi processor case.

\section{PERFORMANCE EVALUATION}

We have implemented the above mentioned algorithms, with the exception of SAMCRA, which we modified from the version provided by F.A. Kuipers into SAMCRA_OP. We also integrated our solution to a PCE in a network with positive preliminary results. In addition, we have done some experiments to empirically determine the behavior of the DORA algorithm; the preliminary results have confirmed the feasibility of using the algorithm as an off-line process within our proposed system.

On the following subsections we test the behavior of the CBR in several simulation scenarios. All simulations have been made on an Intel Centrino Duo laptop with two $2 \mathrm{Ghz}$ processors and $2 \mathrm{~GB}$ of memory.

\subsection{Node scalability}

To test how the response time of the CBR behaves when incrementing the number of nodes in the network, we have

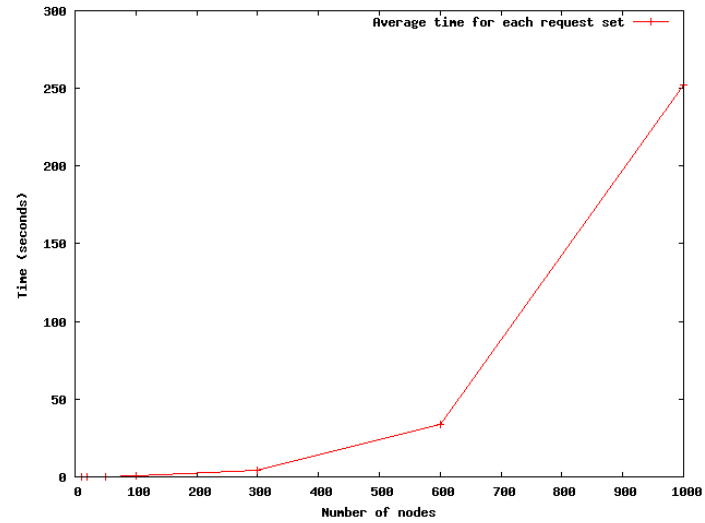

Figure 3: Average time for each path request set on each level

built a series of topologies using the Boston University Representative Internet Topology Generator (BRITE) tool. BRITE builds graphs which can be used as if they were a single autonomous system. In order to simulate a bad case scenario, we have built Waxman topologies with a uniformly distributed node placement, a constant bandwidth of 10 , and two new links per added node. Our experiment levels are $10,20,50,100,300,600$, and 1000 nodes, with 5 topologies per level. Each link has a delay, computed by BRITE as a function of the Euclidean distance between two nodes, as well as two weights, randomVariable1 and randomVariable2, each assigned a weight between 0 and 100 .

For each topology, we have built a series of 20 path request sets, which are sent sequentially to the CBR, with a random source and destination and a constant bandwidth of 5. For each path request set we have requested 10 subpaths, each one of them with a backup path, and a bandwidth of 0.5 for each of the subpaths. Therefore, the total number of paths computed by each path request set is at least 20 , and the total bandwidth is 10 . Finally, we have set very lax constraints for these path requests, setting them at 1000 for delay, and 10000 for each of the random variables. Finally, in our experiments we will optimize our paths to free bandwidth as described in the DORA algorithm without assigning PPV to our links, since we believe that by having the same cost for each link in the constant distribution we can recreate a bad case scenario.

Figure 3 shows the average computation growth rate for each of the path request sets as the number of nodes in the network increases. At first glance, it would appear that the algorithm has a large complexity. However, a closer look shows otherwise. In figure 4 , we show the averaged time for each path request set for the last 10 request sets.

Furthermore, in Figure 5 we show the average time for each of the 20 result sets on the 1000 node topologies.

\subsection{Stricter constraints}

Our second test is to test how the algorithm behaves when the strictness of the constraints increases. We have used the same topologies generated for the previous subsection, but only for the 1000 node level. Our path requests will be the same as the described above, but with only 10 request sets, and one additional constraint: hopcount. We will test how 


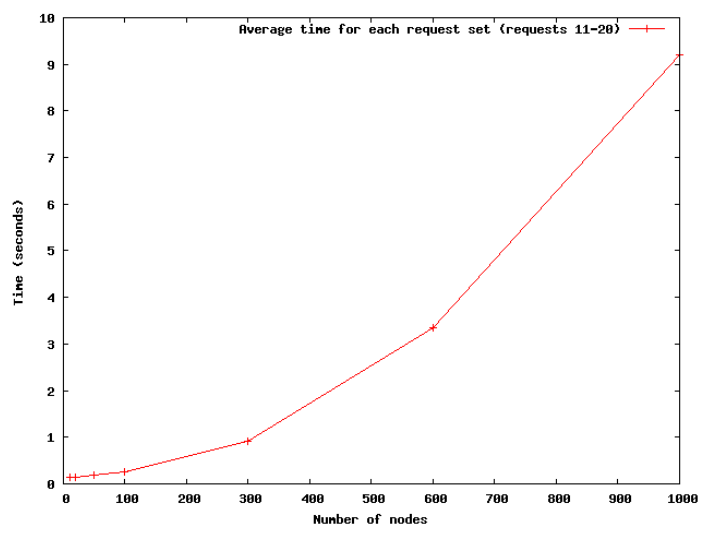

Figure 4: Average time for each path request set on each level, discarding the first ten path requests per level

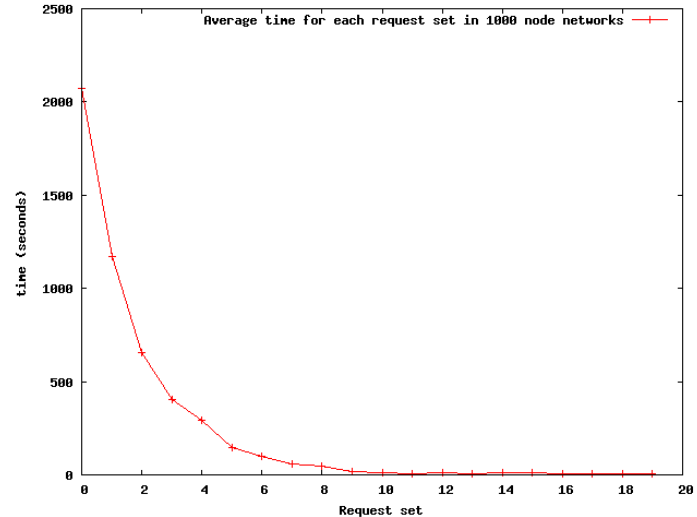

Figure 5: Average time for each of the 20 path request sets in a 1000 node network

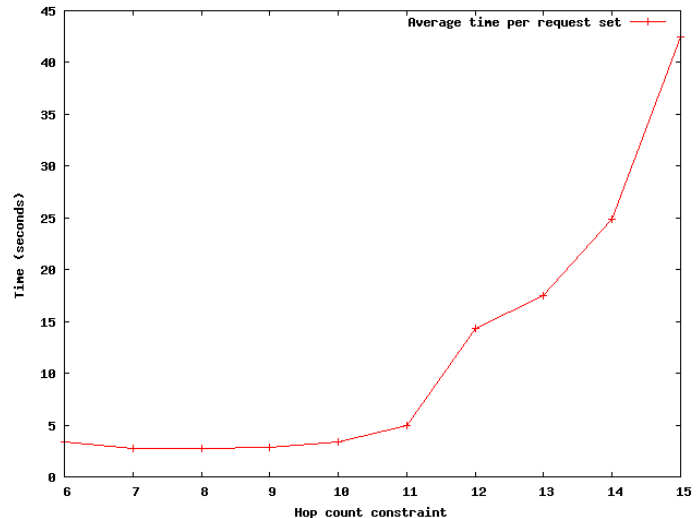

Figure 6: Average time for each request set when the hopcount is constrained in a 1000 node network

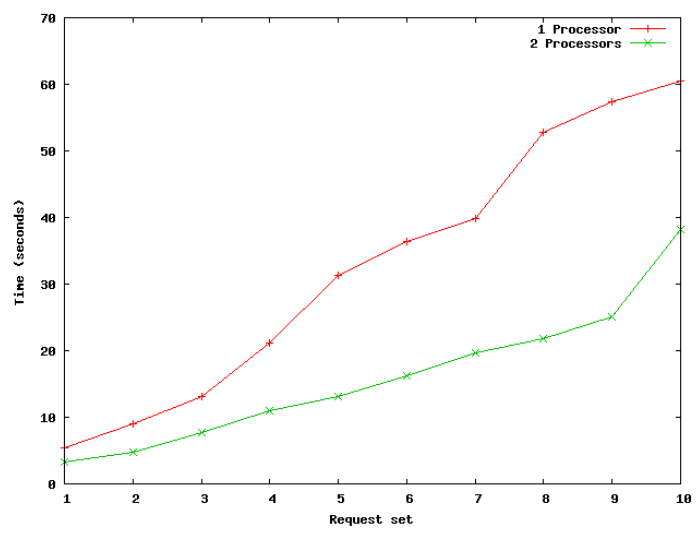

Figure 7: Average time for ten concurrent requests with one process/one processor, and two processes/ two processors

the response time varies when we constrain hopcount to the levels $6,7,8,9,10,11,12,13,14$, and 15 .

On Figure 6, we present the average results for each of the 10 request sets in the 5 topologies. We can see clearly that, for this level, after the hopcount is constrained by 10 hops, the response time starts to grow.

\subsection{Concurrent request scalability}

Our third test aims to show how multiple processors can increase path computation throughput. For this experiment, we have generated 1000 node topologies with uniform bandwidth distribution between 10 and 1024, to better simulate a live network. The rest of the parameters remain as in the first test. Path request sets remain the same, but are reduced to 10 requests per topology, and all 10 are sent concurrently.

Figure 7 presents the time results for the case when one process with one processor serves all requests, and when two processes with two processors serve all requests.

\section{FUTURE WORK}

The use of the exact algorithm SAMCRA has the poten- 
tial limitation that the response time of path computation could in certain cases exceed acceptable values. Furthermore, the present response times could be inadequate for certain applications where path establishment time must be bounded. For those applications, a nonexact algorithm could be the only alternative.

On the other hand, response time for a single request could be reduced with a parallelized MCOP exact algorithm. To our knowledge, no current algorithm for this problem can be easily parallelized.

Finally, as has been discussed on previous sections, there is no exact algorithm that computes backup optimal paths under multiple constraints. The DIMCRA algorithm provides optimal solutions in some scenarios but fails to find a solution or finds suboptimal solutions in other scenarios.

\section{CONCLUSIONS}

Our experiments results show that the SAMCRA_OP algorithm grows in a nonlinear way when the number of nodes in the network increases. However, we can see from Figure 4 that after the first few path requests sets, the computation time drops substantially. The reason is that our chosen topologies have a constant bandwidth, and for the first path requests, every link has the same bandwidth and, therefore, the same cost. The algorithm must therefore try all possibilities within the constraints. But since the constraints are so lax, the possibilities are enormous for 1000 node topologies. Once the first paths are established and their bandwidth reservations made, the cost of the links in those paths rises, and the SAMCRA_OP algorithm can discriminate paths with a higher cost. This is shown in Figure 5 where the average for the first path request set is over 2000 seconds, while the average for the $20^{t h}$ path request set is around 7 seconds.

On our second experiment, we test how the algorithm behaves when the constraints are stricter, for the 1000 node topology. When the number of hops is constrained below 10 , the path computation time appears to be constant, but when the maximum number of hops rises above 10, the computation time starts to rise. It is also interesting that the same topologies that were used for the first experiment were used for the second one, but with much better response times. We can therefore infer, that if the allowed number of hops continues to rise, the response time would be similar to that of the first experiment.

Our third test shows that our proposed scalable architecture would be very useful on large networks with large numbers of requests. This is specially true if we consider that some paths take more time to compute than others, since the constraints, source and destination, optimization variable, backup path and number of subpaths are set by the client. Figure 7 shows an average $33 \%$ decrease on execution time for all 10 simultaneous requests.

As our results show, as long as the network retains certain "differences" that allow the SAMCRA_OP algorithm to choose best paths, or as long as the constraints are not too lax, the CBR scales well for medium-sized networks. Lax constraints, or constraints that many paths will meet, are not very useful, since they are not constraints at all, and should therefore be removed from the path request, which should increase response time.

With the PCE all this computational burden is taken away from routers into an external, dedicated entity. With our in- tegration to the other components of the PCE, the CBR can be used on any medium-sized network to handle the computation of paths subject to multiple constraints. These constraints are not limited to traditional QoS constraints like delay, jitter or loss probability, but can be also constraints that are easier to measure in practice, like monetary cost, hopcount, traffic engineering cost, or any other attribute.

\section{ACKNOWLEDGMENTS}

The first author gratefully acknowledges the financial support of the Red de MacroUniversidades de America Latina through a scholarship of the Programa de Movilidad Universitaria.

\section{REFERENCES}

[1] G. Apostolopoulos, D. Williams, S. Kamat, R. Guerin, A. Orda, and T. Przygienda. QoS Routing Mechanisms and OSPF Extensions. The Internet Engineering Task Force RFC 2676, August 1999

[2] R. Boutaba, W. Szeto, and Y. Iraqi. DORA: Efficient Routing for MPLS Traffic Engineering, Journal of Network and Systems Management, Special Issue on Internet Traffic Engineering and Management, 10(3):309 325, Sept. 2002

[3] S. Cerav and R. Mathar. An Off-Line Traffic Engineering Model for MPLS Networks lcn, p. 0166, 27th Annual IEEE International Conference on Local Computer Networks (LCN'02), 2002.

[4] H. De Neve and P. Van Mieghem. TAMCRA: A Tunable Accuracy Multiple Constraints Routing Algorithm. Computer Communications, Volume 23, Issue 7, Pages 667-679, March 2000

[5] A. Farrel, J.-P. Vasseur, and J. Ash. A Path Computation Element (PCE)-Based Architecture. The Internet Engineering Task Force RFC 4655, August 2006

[6] B Gao, Y Yang, and C Chen. Implementing a Constraint-based Shortest Path First Algorithm in Optical Networks, White Paper for Mahi Networks, can be found at http://suraj.lums.edu.pk/ $\tilde{t e} /$ cspf/Implementing_\%20A_Constraintbased_Shortest_Path_First_Algorithm.pdf, 2003

[7] E. Grampin. Cooperation of Control and Management Plane for the Dynamic Provision of Connectivity Services on MPLS Networks. Doctoral Thesis, Universidad Politecnica de Catalunya, 2001

[8] E. Grampin. PCE Management Interface The Internet Engineering Task Force Internet draft $<$ draft-grampin-pce-mgmt-if-00.txt $>$ Work in progress. Expiration date: January 2006

[9] E. Grampin, J. Serrat, and J. Baliosian. Proposal of a Routing and Management Agent for MPLS networks. Procedings of the Third Iberoamerican Conference on Telematics CITA2003, October 2003

[10] A. Gupta, A. Gupta, B.N. Jain, and S. Tripathi. QoS Aware Path Protection Schemes for MPLS Networks Proceedings of the 15th international conference on Computer communication, 2002

[11] I. Iliadis and D. Bauer. A New Class of Online Minimum-Interference Routing Algorithms 
Proceedings of the Networking Technologies, Services, and Protocols; Performance of Computer and Communication Networks; and Mobile and Wireless Communications: Second International IFIP-TC6

Networking Conference, Pisa, Italy (NETWORKING), May 19-24 2002.

[12] M. Kodialam and T. V. Lakshman. Minimum Interference Routing with Applications to MPLS Traffic Engineering, Proceedings of the Nineteenth Annual Joint Conference of the IEEE Computer and Communications Societies (INFOCOM), 2000.

[13] T. Korkmaz and M. Krunz. Multi-constrained optimal path selection. Proceedings of the Twentieth Annual Joint Conference of the IEEE Computer and Communications Societies. (INFOCOM), 2001

[14] F.A. Kuipers, T. Korkmaz, M. Krunz, and P. Van Mieghem: A Review of Constraint-Based Routing Algorithms. Technical Report, can be found at http://www.nas.its.tudelft.nl/people/ Fernando/papers/TRreviewqosalg.pdf, June 2002.

[15] P. Van Mieghem and F.A. Kuipers. Concepts of Exact QoS Routing Algorithms IEEE/ACM Transactions on Networking (TON) Volume 12, Issue 5, Oct. 2004 Page(s): 851 - 864, October 2004

[16] S. Avallone, F. Kuipers, G. Ventre, and P. Van Miegham. Dynamic Routing in QoS-aware Traffic Engineered Networks. Proc. of EUNICE 2005: Networks and Applications Towards a Ubiquitously Connected World, IFIP WG 6.6, WG 6.4 and WG 6.9 Workshop, Colmenarejo (Spain), published by Universidad Carlos III de Madrid (ISBN: 84-89315-43-4), pp. 222-228, July 6-8 2005

[17] F. Kuipers and P. Van Miegham. The impact of correlated link weights on QoS routing. Proceedings of the Twenty-Second Annual Joint Conference of the IEEE Computer and Communications Societies. (INFOCOM), 2003

[18] Y. Guo, F. Kuipers, and P. Van Miegham. Link-disjoint paths for reliable QoS routing, International Journal of Communication Systems Volume 16, Issue 9, Pages 779-798, November 2003

[19] F. Kuipers. Hop-by-hop Destination Based Routing with Quality of Service Constraints. MSc Thesis, Delft University of Technology, 2000

[20] A. Orda and A. Sprintson. Efficient Algorithms for Computing Disjoint QoS Paths. Proceedings of the Twenty-third Annual Joint Conference of the IEEE Computer and Communications Societies (INFOCOM), 2004

[21] M. Song and S. Sahni. Approximation Algorithms for Multiconstrained Quality-of-Service Routing IEEE Transactions on Computers Volume 55, Issue 5 On page(s): 603-617, May 2006

[22] S. Suri, M. Waldvogel, and P. R. Warkhede. Profile-Based Routing: A New Framework for MPLS Traffic Engineering. Proceedings of the Quality of Future Internet Services: Second COST 263 International Workshop, Coimbra, Portugal, (Qofis), September 24-26, 2001

[23] W Xiao, B.H. Soong, C.L. Law, and Y.L. Guan. Evaluation of Heuristic Path Selection Algorithms for Multi-Constrained QoS Routing. IEEE International
Conference on Networking, Sensing and Control Volume 1 pages 112-116, May 2004 